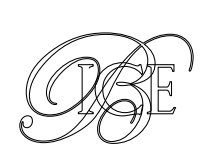

\title{
INSTITUCIONES FINANCIERAS DE DESARROLLO BILATERALES Y BANCOS MULTILATERALES DE DESARROLLO: COMPLEMENTARIEDAD Y EXPERIENCIA/ ACTIVIDAD DE COFIDES EN ESTE ÁMBITO
}

En el contexto actual se constata un crecimiento a nivel mundial de la actividad de las instituciones financieras de desarrollo (IFD) bilaterales, coincidiendo con cambios estructurales en la arquitectura tradicional de financiación del desarrollo. Así, en este artículo se analiza el contexto citado y se describe cómo la actividad de COFIDES se ha ido adaptando al mismo a través de diferentes fórmulas innovadoras, entre las que destacan la puesta en valor de sus treinta años de liderazgo en este ámbito, la captación de nuevos recursos financieros, un mayor apoyo a los instrumentos de financiación combinada o blended finance de la UE y, complementariamente, una mayor integración a los estándares internacionales compartidos por las IFD europeas en cuestiones sociales y ambientales. Todo ello para seguir apoyando al sector privado en su crecimiento vía inversión directa en el exterior (IDE) en países en desarrollo.

Palabras clave: blending, Unión Europea, financiación del desarrollo, sector privado, instituciones multilaterales.

Clasificación JEL: F63, G23.

\section{Introducción}

Como institución financiera de desarrollo (IFD), la actividad de COFIDES tiene como principal objetivo la financiación de proyectos de inversión directa en el exterior (IDE) a través del sector privado.

Como otras IFD, COFIDES opera con criterio de rentabilidad, y su actividad principal consiste

*Presidente y CEO de COFIDES, SA, SME. Versión de abril de 2018.

En la preparación de este trabajo agradezco la colaboración de Raúl Moreno, Gerente-Coordinador de COFIDES. en facilitar financiación a proyectos privados viables y sostenibles de inversión en el exterior. COFIDES cuenta con un doble mandato estatutario que exige que sus operaciones contribuyan tanto al desarrollo de los países receptores de las inversiones, como a la internacionalización de la economía y de las empresas españolas.

Para cumplir con esta doble finalidad, utiliza sus propios recursos para financiar proyectos de inversión en países emergentes o en desarrollo y, por otro lado, gestiona en exclusiva y por cuenta del Estado (Secretaría de Estado $D$ 
de Comercio, Ministerio de Economía, Industria y Competitividad) los fondos FIEX (Fondo para Inversiones en el Exterior) y FONPYME (Fondo para Operaciones de Inversión en el Exterior de la Pequeña y Mediana Empresa), creados para financiar proyectos de inversión en el exterior con independencia del grado de desarrollo del país destino del proyecto. Además, COFIDES da apoyo al Ministerio de Asuntos Exteriores y de Cooperación en la gestión del Fondo para la Promoción del Desarrollo (FONPRODE) en operaciones de financiación reembolsable y, desde 2016, puede gestionar recursos del presupuesto de la UE para financiación combinada o blending ${ }^{1}$.

En total, COFIDES posee una capacidad de movilización de recursos de cerca de $2.500 \mathrm{mi}$ llones de euros (Marín-Hernández, 2013 y 2015).

COFIDES actúa siempre bajo un criterio de riesgo compartido, según el cual aporta recursos de forma conjunta con el sector privado para las inversiones ligadas a cada proyecto $y$, por tanto, moviliza también los recursos privados a través de la cofinanciación; pudiendo contribuir financieramente a través de un amplio rango, en principio, con importes comprendidos entre 75.000 euros y 30 millones de euros por proyecto.

Esta actividad, al igual que la del resto de las IFD bilaterales, genera una serie de efectos positivos, tanto en el país de origen, vía fortalecimiento de la matriz y/o generación/mantenimiento de empleo de calidad, como en los países destinatarios de IDE, que además contribuyen a

\footnotetext{
1 La financiación combinada o blending es un instrumento de financiación de la Unión Europea que se articula mediante la combinación de subvenciones de la Unión Europea con financiación (vía préstamo o capital) de entidades financieras públicas y privadas acreditadas (como es el caso de COFIDES). El destinatario de la financiación del blending (sector público o privado) se beneficia de condiciones concesionales gracias a la aportación de la Comisión Europea, que puede ser en forma de: 1) reducción del coste (donación para inversión; subvención tipo interés), 2) reducción del riesgo (primeras pérdidas; garantías) o 3) mejora de la calidad (asistencia técnica).
}

la consecución de los objetivos marcados por las políticas e iniciativas de financiación del desarrollo tanto nacionales como internacionales. Estos efectos positivos de la actividad inversora en el exterior a través del sector privado suelen agruparse en: 1) efectos de tipo cuantitativo, como la creación de empleo y la generación de ingresos fiscales, y 2) efectos de tipo cualitativo, como la transferencia de tecnología y conocimiento o la innovación (Mendoza et al.; COFIDES/ESADE, 2018).

En su puesta en marcha y ejecución ha de destacarse la utilización de exigentes procedimientos de due dilligence financiera, medioambiental y social que garantizan el respeto y alineamiento con los más altos estándares internacionales.

En este artículo se analizará, en primer lugar, el contexto internacional actual en el que se desarrolla la actividad de las IFD, incluyendo un análisis de la nueva agenda de financiación internacional del desarrollo.

En segundo lugar, se describirá cómo la actividad de COFIDES se ha ido adaptando a este nuevo entorno a través de diferentes fórmulas innovadoras, entre las que destacan la captación de nuevos recursos financieros procedentes de terceros, un mayor apoyo a los instrumentos de financiación combinada o blending de la UE y, complementariamente, una mayor integración de la compañía en los usos y estándares internacionales compartidos por las IFD europeas en cuestiones sociales y ambientales.

\section{La nueva Agenda de Desarrollo post 2015 y el papel de las IFD}

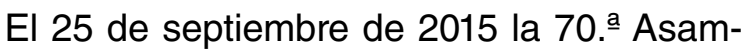
blea General de Naciones Unidas (AGNU) $\triangleright$ 
CUADRO 1

COMPARATIVA DE LAS IFD BILATERALES

\begin{tabular}{|c|c|c|c|c|}
\hline IFD & Sectores & Área geográfica & Instrumentos & Actividad ligada \\
\hline BIO (Bélgica) & SF, PME, I & AF, AP, LA, OM & AF, AP, LA, OM & No \\
\hline CDC (Reino Unido) & SF, I & AF, SA & $\mathrm{C}, \mathrm{CC}, \mathrm{P}$ & No \\
\hline COFIDES (España) & I, AG, M, PME, S & M, LA & $\mathrm{C}, \mathrm{CC}, \mathrm{P}$ & Sí \\
\hline DEG (Alemania) & I, AG, M, PME, SF & M & $\mathrm{C}, \mathrm{CC}, \mathrm{P}$ & No \\
\hline FINNFUND (Finlandia) & $S F, I, A G$ & M & $\mathrm{C}, \mathrm{CC}, \mathrm{P}$ & Sí \\
\hline FMO (Países Bajos) & SF, I, AG & M & $\mathrm{C}, \mathrm{CC}, \mathrm{P}, \mathrm{G}$ & No \\
\hline IFU (Dinamarca) & $S F, I, A G$ & M & $\mathrm{C}, \mathrm{CC}, \mathrm{P}, \mathrm{G}$ & Sí \\
\hline NORFUND (Noruega) & $S F, I, A G$ & $A F, L A, A P$ & $\mathrm{C}, \mathrm{CC}, \mathrm{P}, \mathrm{G}$ & No \\
\hline OeEB (Austria) & SF, I, AG & M & $\mathrm{C}, \mathrm{CC}, \mathrm{P}, \mathrm{G}$ & No \\
\hline OPIC (Estados Unidos) & I, SF, AG, PME & M & $P, G, S$ & Sí \\
\hline PROPARCO (Francia) & $S F, I, A G, S$ & $M$ & $\mathrm{C}, \mathrm{CC}, \mathrm{P}$ & No \\
\hline SBI (Bélgica) & $A G, I, S$ & $A P, L A, A F$ & $\mathrm{C}, \mathrm{CC}, \mathrm{P}$ & Sí \\
\hline SIFEM (Suiza) & SF & M & $\mathrm{C}, \mathrm{CC}, \mathrm{P}$ & No \\
\hline SIMEST (Italia) & I, AG, S & M & $\mathrm{C}, \mathrm{CC}, \mathrm{P}$ & Sí \\
\hline SOFID (Portugal) & I, AG, S & AP, LA, OM & $P, G$ & Sí \\
\hline SWEDFUND (Suecia) & SF, I, AG & M & $\mathrm{C}, \mathrm{CC}, \mathrm{P}, \mathrm{G}$ & No \\
\hline \multicolumn{5}{|c|}{$\begin{array}{l}\text { Áreas geográficas: } M=\text { mundial; } A F=\text { África; } A P=\text { Asia Pacífico; } S A=\text { Sudeste Asiático; } E E=\text { Europa del Este; } L A=\text { Latinoamérica; } \\
\mathrm{OM}=\text { Oriente Medio y norte de África. }\end{array}$} \\
\hline \multicolumn{5}{|c|}{ Instrumentos: $\mathrm{P}=$ préstamos; $\mathrm{G}=$ garantías; $\mathrm{S}=$ seguros; $\mathrm{C}=$ capital; $\mathrm{CC}=$ cuasicapital. } \\
\hline \multicolumn{5}{|c|}{ Fuente: elaboración propia con datos de la European Association of Development Finance Institutions (EDFI). } \\
\hline
\end{tabular}

dio por concluida la Agenda de los Objetivos de Desarrollo del Milenio (ODM), reemplazándola por la Agenda de Desarrollo post 2015 con un horizonte temporal de quince años. Tras un proceso participativo en el que, entre otros, colaboraron aproximadamente 1.500 empresas a través de la red Pacto Mundial, se establecieron los nuevos Objetivos de Desarrollo Sostenible (ODS), para cuya consecución hasta 2030 se exigirán más recursos financieros. En este contexto de mayor exigencia a toda la comunidad internacional y tras la Conferencia de Addis Abeba (2015) y su Plan de Acción, se otorga al sector privado un papel protagonista en la financiación del desarrollo a nivel mundial.

A diferencia de lo sucedido en las conferencias anteriores de Monterrey (2002) y Doha (2008), en el Plan de Acción aprobado en 2015 por primera vez se pone el énfasis no solo en la financiación concesional, sino también en la IDE, en las colaboraciones público-privadas e incluso en el aumento de la recaudación fiscal en los países en desarrollo?

Bajo este enfoque con una clara interrelación entre los ámbitos públicos y privados, la oportuna coordinación entre las iniciativas de IDE y la financiación otorgada por los bancos multilaterales de desarrollo (MDB) y las IFD será clave en los próximos años (CSIS, 2016).

En lo relativo a la cuantificación del nivel de recursos necesarios para la consecución de la Agenda 2030, si bien no existen datos concluyentes, de acuerdo con estimaciones de la $\triangleright$

2 En el punto 36 de la Agenda de Acción de Addis Abeba se subraya la relevancia de conciliar los incentivos de los sectores públicos y privados. 
CUADRO 2

ESTRATEGIAS COMPLEMENTARIAS DE FINANCIACIÓN DEL DESARROLLO

\begin{tabular}{|c|c|c|}
\hline $\begin{array}{c}\text { AYUDA OFICIAL } \\
\begin{array}{c}\text { Subvenciones y cooperación técnica } \\
\text { con fines humanitarios o asistencia } \\
\text { al desarrollo }\end{array}\end{array}$ & $\begin{array}{c}\text { CRÉDITOS A ESTADOS } \\
\text { Préstamos reembolsables de carácter } \\
\text { tanto concesional como no concesional } \\
\text { dirigidos a Estados o instituciones } \\
\text { públicas }\end{array}$ & $\begin{array}{c}\text { SECTOR PRIVADO } \\
\text { Operaciones de capital, cuasicapital, } \\
\text { préstamos y/o garantías dirigidas a } \\
\text { proyectos privados sostenibles y } \\
\text { comercialmente viables }\end{array}$ \\
$\begin{array}{c}\text { AGENCIAS DE COOPERACIÓN AL } \\
\text { DESARROLLO }\end{array}$ & $\begin{array}{c}\text { BANCOS DE DESARROLLO (MDB) } \\
\text { INSTITUCIONES FINANCIERAS } \\
\text { DE DESARROLLO (IFD) }\end{array}$ \\
\hline \multicolumn{2}{|c|}{$\begin{array}{c}\text { BLENDED FINANCE } \\
\text { Combinación de subvenciones con financiación (vía préstamo o capital) de entidades financieras públicas y privadas }\end{array}$} \\
\hline Fuente: European Association of Development Finance Institutions (EDFI).
\end{tabular}

Red de Soluciones para el Desarrollo Sostenible (SDSN por sus siglas en inglés), establecida en 2012 por la ONU, la financiación que requerirán los ODS es de dos a tres mil millones de dólares anuales (alrededor del 4 por 100 del PIB mundial), cifra que podría ascender a siete mil millones anuales si tenemos en cuenta la necesidad de financiación en el sector de infraestructuras.

La Ayuda Oficial al Desarrollo (AOD) de los Estados donantes del Comité de Ayuda al Desarrollo (CAD) de la OCDE se situó en 2015 en 131.586 millones de dólares. A pesar de que se ha incrementado en torno a dos tercios en términos reales desde el año 2000, es una cifra que parecería aún insuficiente. Por otro lado, el impacto de la crisis económica en los donantes tradicionales hace pensar que el volumen de las contribuciones en $A O D$ de los países desarrollados no se incrementará sustancialmente durante los próximos años. Por todo ello, resulta clara la necesidad de involucrar a otros actores a la hora de financiar los ODS.

\section{El contexto internacional actual y su impacto en las IFD}

Otro rasgo característico de la financiación del desarrollo durante las dos últimas décadas ha sido, probablemente, el de la diferente estructura de pesos relativos de la AOD dentro del conjunto de instrumentos que habitualmente han sido utilizados por los financiadores. Esto se ha concretado en una tendencia hacia un menor nivel del peso relativo de la AOD, pero coincidiendo, a su vez, con un aumento considerable de los flujos privados de inversión y con un crecimiento notable de las IFD, tanto bilaterales como multilaterales (MDB).

Al mismo tiempo, esta evolución durante aproximadamente los últimos veinticinco años se ha visto acompañada de una reducción relevante de las tasas de pobreza a nivel mundial (Norberg, 2017). Además, ha llevado aparejada consigo la necesidad de abordar nuevos desafíos globales que requieren actualmente un mayor esfuerzo entre todos los actores involucrados y una mayor necesidad de utilizar eficientemente los recursos disponibles para ejecutar las diferentes políticas de cooperación al desarrollo.

Bajo estas circunstancias, las IFD se están volviendo aún más complementarias para estas políticas, tanto por su experiencia y liderazgo en este ámbito como por la necesidad de incrementar la eficiencia y otros efectos adicionales de la ayuda tradicional vía AOD.

Por ello, los policy makers nacionales e internacionales están teniendo este elemento cada vez más en cuenta en sus estrategias $D$ 
y así se refleja en la actual agenda de financiación del desarrollo, en la que el aumento del peso y relevancia otorgado al sector privado es creciente y un elemento clave para hacer realidad la aspiración de apalancar el máximo de recursos pasando de «millones a trillones» disponibles ${ }^{3}$.

El aumento de la financiación disponible para inversiones en países y economías en desarrollo coincide también con la consolidación y mayor protagonismo de nuevos actores como, por ejemplo, los flujos privados procedentes de la filantropía, las remesas de emigrantes, las nuevas economías emergentes a través sus propios bancos de desarrollo (Asian Infrastructure Development Bank, New Development Bank) e incluso el aumento de los recursos propios de muchos países en desarrollo.

Un aspecto fundamental que ha de valorarse en el escenario descrito es el de la necesidad de continuar haciendo esfuerzos en el objetivo final de ajustar la disponibilidad de financiación y la inversión requerida por los países en desarrollo. En la última década, los países en desarrollo han disfrutado de un mayor y mejor acceso a los mercados internacionales de capital, pero aún sigue siendo muy necesario seguir trabajando por la búsqueda de equilibrio entre los recursos disponibles y la capacidad de absorción de los mismos. Así, las IFD y los MDB han identificado el acceso a la financiación como un obstáculo importante para el crecimiento del sector privado en los países en desarrollo. Persisten ciertos fallos de mercado que impiden una conexión eficiente entre los grandes fondos de

3 From Billions to Trillions: Transforming Development Finance Post2015 Financing for Development: Multilateral Development Finance, elaborado conjuntamente por el Banco Africano de Desarrollo, el Banco Asiático de Desarrollo, el Banco Europeo de Reconstrucción y Desarrollo, el Banco Europeo de Inversiones, el Banco Interamericano de DesarroIlo, el Fondo Monetario Internacional y el Grupo Banco Mundial para la reunión del Development Committee del 18 de abril de 2015. ahorro en países desarrollados, en particular aquellos participados por inversores institucionales de largo plazo como los fondos de pensiones, con las oportunidades de inversión en los mercados de países y economías en desarrollo (Kleiterp, 2016).

Es, en este contexto, donde entidades como los MBD y las IFD cuentan con la capacidad de aportar valor $y$, por tanto, ofrecer soluciones a este tipo de desajustes a través de los instrumentos que entidades como COFIDES vienen utilizando desde hace treinta años.

En las economías desarrolladas la asistencia pública al desarrollo se canaliza, mayoritariamente, a través de tres diferentes modalidades. En primer lugar, a través de agencias de cooperación que, principalmente, conceden subvenciones de forma directa o indirecta, canalizando de esta forma la ayuda al desarrollo y la asistencia humanitaria. En segundo lugar, a través de la participación en bancos de desarrollo multilaterales con participación pública. En último lugar, las IFD bilaterales, que proporcionan productos financieros y de asesoramiento especializados dirigidos al sector privado que invierte en países y economías en desarrollo.

Por tanto, podemos colegir, dada la realidad actual imperante, que las IFD bilaterales se han convertido en un tercer pilar, importante, que complementa a los instrumentos de cooperación tradicionales y a los bancos multilaterales. En los últimos años su relevancia se ha incrementado notablemente. En 2002, las IFD invirtieron aproximadamente 10.000 millones de dólares a nivel mundial en el sector privado, o alrededor de una sexta parte de la AOD. Según un estudio del Centro de Estudios Estratégicos e Internacionales (CSIS, 2016) en colaboración con EDFI, se estima que los compromisos totales de las IFD ascendieron a $\triangleright$ 
CUADRO 3

CARTERA COMPROMETIDA DE COFIDES EN PAÍSES Y ECONOMÍAS EN DESARROLLO

\begin{tabular}{|l|r|}
\hline \multicolumn{1}{|c|}{ Países/economías } & \multicolumn{1}{c|}{ Euros } \\
\hline África & 29.956 .227 \\
Sudeste Asiático y Pacífico & 86.880 .568 \\
Europa y Asia Central & 69.881 .506 \\
Latinoamérica y Caribe & 440.024 .436 \\
Oriente Medio y norte de África & 16.184 .641 \\
Asia & 101.056 .627 \\
Financiación corporativa & 136.586 .196 \\
Total & $\mathbf{8 8 0 . 5 7 0 . 2 0 1}$ \\
\hline Fuente: elaboración propia. & \\
\hline
\end{tabular}

casi 70.000 millones de dólares en 2014, prácticamente la mitad de la AOD de ese año (Runde et al., 2016). A nivel europeo, de 2003 a 2015 la cartera consolidada de las quince entidades miembros de la Asociación Europea de IFD (EDFI) aumentó un 260 por 100, desde los 10.000 hasta los 36.000 millones de euros.

Las IFD sostienen que la creación de empleo es el canal principal a través del cual el crecimiento económico se traslada a las economías en desarrollo, generando efectos positivos en las poblaciones más desfavorecidas. Se estima que 9 de cada 10 puestos de trabajo (EDFI, 2016) son creados por el sector privado en los países de renta baja y media.

En el particular discurso de las IFD sobre la financiación del desarrollo, el segundo elemento clave para entender el papel del sector privado es el de los ingresos fiscales generados en las economías receptoras de inversiones, por su papel catalizador de ingresos fiscales propios (recursos nacionales) en las economías receptoras de IDE.

Por tanto, por la relevancia creciente de la IDE frente a la AOD, así como por el progresivo peso de los recursos nacionales, asistimos actualmente a un protagonismo mayor de las IFD que contribuyen positivamente a la creación de empleo y la generación de ingresos fiscales en destino.

En este sentido, la cartera comprometida total correspondiente a 2017 (países en desarrollo y países desarrollados) gestionada por COFIDES ascendió a 1.051.773.717 euros. Por lo que, en línea con lo comentado, vemos que el 83,7 por 100 de la financiación gestionada por COFIDES se dirigió a países en desarrollo (Cuadro 3).

\section{Los acuerdos de cofinanciación de COFIDES con bancos multilaterales de desarrollo (MDB)}

En la actualidad COFIDES cuenta con una capacidad de intervención global, a través de los mecanismos e instrumentos financieros en los que participa, de cerca de 2.500 millones de euros, pudiendo invertir hasta 30 millones por proyecto. Desde su creación, COFIDES ha aprobado más de 910 proyectos de inversión en cerca de 90 países, en los que ha comprometido recursos por importe superior a 3.000 millones de euros para una inversión superior a 34.900 millones de euros.

Esta evolución en el crecimiento operativo y financiero de la compañía ha venido acompañada al mismo tiempo de un crecimiento y mayor especialización en el control de los riesgos no financieros y un mayor alineamiento con determinados estándares internacionales en los ámbitos medioambiental, social y de desarrollo. COFIDES vela por el cumplimiento de estos por parte de las compañías financiadas y cautela la sostenibilidad de sus operaciones desde un triple enfoque: sostenibilidad económica, medioambiental y social (Marín-Hernández, 2013 y 2015).

En el contexto descrito, la estrategia de COFIDES se basa actualmente en tres ejes $D$ 
complementarios: fortalecimiento, experiencia acumulada y puesta en valor de los recursos tradicionales (recursos propios, FIEX y FONPYME), la obtención de recursos financieros de nuevas fuentes adicionales con una especial atención a la utilización de la financiación combinada (blending) y la aplicación de los más exigentes estándares internacionales en su operativa interna en los ámbitos mencionados.

En octubre de 2017 y en marzo de 2018, dando continuidad a esta estrategia, COFIDES ha suscrito importantes acuerdos de cofinanciación con dos instituciones financieras de desarrollo de relevancia: el Grupo Banco Interamericano de Desarrollo, a través de su subsidiaria especializada en sector privado (BID INVEST) y el Banco Europeo de Inversiones (BEI).

En el primer caso (BID INVEST), el acuerdo (denominado en su versión original CoFinancing Framework Agreement) establece un marco procedimental para la formalización y puesta en marcha de propuestas de cofinanciación de proyectos de inversión entre BID INVEST y COFIDES. El acuerdo se inspira en la denominada modalidad one-way, que contempla la operativa de que BID INVEST sea la entidad proponente de operaciones potenciales ${ }^{4}$. Este tipo de acuerdos había sido utilizado por el grupo BID con anterioridad con otras IFD europeas como FMO (Países Bajos) y PROPARCO (Francia). Una de las posibilidades que permitirá el acuerdo es la de utilizar recursos conjuntos para iniciativas de financiación combinada (blending), que podría liderar COFIDES en calidad de IFD bilateral europea acreditada ante la UE en la región de Latinoamérica ${ }^{5}$.

\footnotetext{
4 No obstante, en la práctica ya iniciada, es habitual que también se proceda a la presentación/discusión de posibles proyectos propuestos en la dirección inversa, es decir, propuestos desde COFIDES.

5 EI BID también es una entidad acreditada ante la UE, pero, al ser un banco de desarrollo regional no europeo, de momento solo puede liderar
}

En cuanto a los aspectos estrictamente procedimentales, el acuerdo contempla una fase preliminar para la aprobación de operaciones denominada «valoración previa» o «appraisal process», que se aplicará siempre para cada propuesta de operación y podrá ser simultánea al procedimiento de due dilligence. En la práctica, si esta fase preliminar obtiene una valoración positiva, se traduce en una invitación formal de la entidad proponente (BID INVEST) a la IFD (COFIDES) para analizar la viabilidad de cofinanciación de una determinada operación. En cuanto a la fase de due dilligence prevista, esta se llevará a cabo por BID INVEST y analizará tanto la viabilidad del proyecto de inversión como la idoneidad del futuro prestatario (borrower) y/o promotor privado (sponsor) en los ámbitos financiero, jurídico, comercial, técnico, medioambiental, social, corporativo, fiscal, laboral y cualquier otro que se considere aplicable y relevante en cada caso.

Con las debidas cautelas de confidencialidad, la IFD (COFIDES) podrá participar en fase de due dilligence a través de la consulta de los documentos de trabajo, de los informes de consultoría (si los hubiera) y de las valoraciones de BID INVEST sobre la operación, incluyendo la posibilidad de trasladar comentarios o recomendaciones. La puesta en marcha de la cofinanciación se llevará a cabo previa aceptación formal por parte de la IFD (COFIDES) mediante una Letter of Interest emitida en un plazo no superior a 21 días desde la invitación formal de la entidad proponente (BID INVEST) y formalizada mediante una Letter of Approval entre ambas, que se acompañará del Terms Sheet (Documento de Condiciones) suscrito con el cliente prestatario (borrower).

proyectos en el Caribe, en aplicación de los acuerdos alcanzados a este respecto. 
Por último, las relaciones bilaterales entre BID INVEST y COFIDES, como cofinanciadores, se regularán para cada operación en un documento contractual separado (Intercreditor Agreement) que recogerá los derechos y obligaciones de cada parte, las cantidades aportadas por cada parte y los tipos de interés y resto de condiciones financieras aplicables en cada operación.

En el caso del acuerdo con el BEI (denominado en su versión original «Collaboration and Co-financing Framework»), se trataría del primer acuerdo de este tipo firmado por el Banco de la Unión Europea con una IFD bilateral europea. Se dirige principalmente a facilitar la cofinanciación de operaciones en el marco de las facilidades de blending de la UE y del Fondo Verde para el Clima de la Convención Marco de Naciones Unidas sobre el Cambio Climático (CMNUCC) ${ }^{6}$ y cuenta con unos recursos máximos asignados por un monto de 200 millones de euros que permitirán financiar operaciones durante un periodo de tres años.

En este caso se contempla que COFIDES lidere estas operaciones ante la Unión Europea (o, en su caso, ante el Fondo Verde para el Clima), en calidad de entidad acreditada. Asimismo, desde un punto procedimental, COFIDES será competente para la gestión integral de los proyectos en todas sus fases (appraisal, due dilligence, ejecución, seguimiento y evaluación ex post).

En principio, el Acuerdo de Cofinanciación con el BEl estipula que las operaciones de inversión tipo sean de un máximo de 25 millones de euros, pudiendo el BEI en estos casos utilizar de forma directa la due dilligence, estudios previos y análisis preparatorios llevados a cabo por COFIDES.

6 A la fecha de cierre de este artículo, COFIDES está en la última fase del proceso de acreditación del Fondo Verde.
Como se mencionaba al inicio de este epígrafe, el tercer eje estratégico y complementario a la actividad financiera es el de la creciente coordinación y alineamiento a nivel internacional de los estándares utilizados por las IFD en los procesos de análisis, formalización y seguimiento de operaciones.

En este sentido, COFIDES ha integrado en sus políticas internas una serie de estándares que potencian esta integración y facilita la puesta en marcha de acuerdos como los citados. Entre las iniciativas en las que COFIDES ha participado activamente y que forman parte de sus procedimientos internos destacan especialmente las siguientes:

- COFIDES aplica la Declaración de Principios de Financiación Responsable (Consenso de Roma $)^{7}$ que consagran el respeto por los derechos humanos y la sostenibilidad medioambiental como requisitos vinculados a los proyectos de inversión en los que participa financieramente.

- En el ámbito de la financiación combinada (blended finance), COFIDES aplica las Guidelines on Blended Concessional Finance for Private Sector Projects ${ }^{8}$, impulsadas desde 2013 por un grupo representativo de las IFD y los $\mathrm{MDB}^{9}$ y que consagran la aplicación de cinco principios básicos para esta modalidad de financiación (adicionalidad, crowing-in del sector privado, sostenibilidad económica, $D$

\footnotetext{
7 https://www.edfi.eu/wp/wp-content/uploads/2017/10/EDFIPrinciples-for-Responsible-Financing.pdf

8 https://www.edfi.eu/wp/wp-content/uploads/2017/10/DFl-BlendedConcessional-Finance-for-Private-Sector-Operations_Summary-R....pdf

9 Iniciativa suscrita en octubre de 2017 por International Finance Corporation (IFC), African Development Bank (AfDB), Asian Development Bank (AsDB), Asian Infrastructure Investment Bank (AllB), European Bank For Reconstruction and Development (EBRD), European Development Finance Institutions (EDFI), European Investment Bank (EIB), Inter-American Development Bank Group (IDBG), Islamic Corporartion for the Development of the Private Sector (ICD).
} 
CUADRO 4

RESUMEN DE ESTÁNDARES DE COFIDES

\begin{tabular}{|c|}
\hline Ámbitos financiación responsable y desarrollo \\
\hline Principios de Financiación Responsable (Consenso de Roma) \\
\hline Guidelines on Blended Concessional Finance for Private Sector Projects \\
\hline Signatario de la iniciativa «Harmonized Indicators for Private Sector Operations» \\
\hline Política Desarrollo COFIDES \\
\hline Metodología GPR de medición de Efectos en Desarrollo \\
\hline Ámbitos social, laboral, derechos humanos y anticorrupción \\
\hline Declaración Universal de Derechos Humanos (Naciones Unidas) \\
\hline Convención para la Eliminación de todas las Formas de Discriminación Contra la Mujer (Naciones Unidas) \\
\hline Convenios Fundamentales de la Organización Internacional del Trabajo (OIT) \\
\hline Convenios Relativos al Trabajo Decente (OIT) \\
\hline Convención sobre los Derechos del Niño (Naciones Unidas) \\
\hline Principios del Pacto Mundial (Naciones Unidas) \\
\hline Líneas Directrices para Empresas Multinacionales (OCDE) \\
\hline Ámbitos medioambiental y social \\
\hline Lista de Actividades y Sectores Excluidos de Financiación, en línea con IFC (Política Medioambiental y Social de COFIDES) \\
\hline
\end{tabular}

refuerzo del mercado y la promoción de altos estándares socioambientales).

- Para valorar los efectos en desarrollo de las inversiones, COFIDES establece un diálogo activo con el sector privado. Los resultados se reflejan en un rating de efectos en desarrollo propio (basado en la herramienta GPR $®$ del grupo público alemán DEG-KfW), tanto de forma previa a la puesta en marcha de los proyectos de inversión como periódicamente una vez formalizada la financiación de estos.

- Como signatario de la iniciativa HIPSO ${ }^{10}$ (Harmonized Indicators for Private Sector Operations), en la que participan veinticinco IFD, COFIDES utiliza un conjunto de indicadores armonizados en todos sus análisis de efectos en desarrollo.

- COFIDES incorpora altos estándares en los ámbitos laboral, de protección de los

10 https://indicators.ifipartnership.org/indicators/ derechos humanos, de igualdad de la mujer, así como medidas anticorrupción, incorporando las mejores prácticas internacionales a las relaciones contractuales con sus clientes.

- COFIDES presenta un alto grado de compromiso con el Gobierno Corporativo, verificación de su memoria de sostenibilidad dentro de los más amplios estándares internacionales, así como toda la comunicación de su información financiera y no financiera; habiendo además recientemente iniciado los trámites para la edición de su primer informe integrado.

- Por último, en línea con la mayoría de las IFD y los MBD, COFIDES aplica una lista de actividades excluidas, en línea con el enfoque de la Corporación Financiera Internacional (IFC, Grupo Banco Mundial), que forma parte de su política interna medioambiental y social. 


\section{Conclusiones}

En el contexto actual, se constata un crecimiento a nivel mundial de la actividad de las IFD bilaterales. Este crecimiento coincide con cambios estructurales en la arquitectura tradicional de financiación del desarrollo, que exigen contar con mayores recursos y una mayor presencia en nuevas regiones para atender los nuevos desafíos de la agenda internacional. Es igualmente importante continuar con el esfuerzo de integración y armonización a nivel internacional en las metodologías de medición de efectos en desarrollo, en el seguimiento de los ODS y en los estándares socioambientales utilizados en los procedimientos de due dilligence internos.

Ante este nuevo contexto, el papel del sector privado será clave para alcanzar tanto los ODS como buena parte de los objetivos estratégicos de la cooperación internacional al desarrollo.

Para las IFD será necesario además potenciar las iniciativas de financiación combinada, como vía para apalancar un mayor nivel de recursos financieros y ser capaz de hacer más con menos recursos públicos disponibles.

COFIDES, líder y referencia en su sector en este ámbito, y con treinta años de experiencia en el mismo, está intensificando su relación estratégica con los MDB y otras instituciones financieras multilaterales, por su relevancia en la nueva arquitectura de financiación del desarrollo y como forma de acceso, con criterio de especialización y complementariedad, a recursos financieros adicionales que permitirán incrementar la cartera de operaciones sostenibles de IDE a través del sector privado. Así, junto a la gestión integral y puesta en valor de las aportaciones positivas al desarrollo vía internacionalización del sector privado español a través de sus recursos propios, fondos FIEX y FONPYME, que han supuesto apoyar más de 910 proyectos en cerca de 90 países y por importes superiores a 34.900 millones de euros de inversión con más de 3.000 millones de euros de apoyo financiero, se han unido, en una clara estrategia de diversificación y crecimiento ordenado de la compañía, la acreditación ya obtenida desde 2016 para poder gestionar recursos del presupuesto de la UE vía blending, la presentación para poder obtener la misma posibilidad en relación al Fondo Verde del Clima - que esperamos obtener en breve una vez pasados todos los exigentes requerimientos-, así como los acuerdos importantes con BID INVEST y BEI. Sin duda, crecer de forma ordenada y, además, acompañado de instituciones de prestigio y relevancia, hacen que COFIDES dé un paso más de liderazgo positivo en su ámbito de actuación, muy útil para el sector privado nacional en sus estrategias de crecimiento vía IDE en países en desarrollo.

\section{Bibliografía}

[1] CENTER FOR STRATEGIC AND INTERNATIONAL STUDIES -CSIS- (2016). Development Finance Institutions Come of Age. Policy Engagement, Impact, and New Directions. CSIS.

[2] EUROPEAN DEVELOPMET FINANCE INSTITUTIONS-EDFI-(2016).Investing to Create Jobs, Boost Growth and Fight Poverty. EDFI.

[3] KLEITERP, N. y WIERSMA, M. (2017). Banking for a Better World. FMO and Amsterdam University Press.

[4] MARÍN-HERNÁNDEZ, S. (2013). «COFIDES y sus específicas características en la financiación internacional». Revista de Economía ICE, n.. 873, pp. 83-106.

[5] MARÍN-HERNÁNDEZ, S. (2015). «Financiación para el Desarrollo desde el ámbito de la internacionalización: productos e $D$ 
información financiera». Revista de $A E C A, \mathrm{n} . \stackrel{\circ}{ }$ 111, pp. 49-52.

[6] MARÍN-HERNÁNDEZ, S. (2015). «Estructura, evolución y valor añadido de la Compañía Española de Financiación del Desarrollo (COFIDES)». Revista de Economía ICE, n.ㅇ 882, pp. 69-93.

[7] MENDOZA MAYORDOMO, X.; IMBERNÓN SÁINZ, A.; PEREDA MACIAS, A.; MARIN-
HERNÁNDEZ, S. et al. (2017). La inversión directa de las empresas españolas en el exterior. Efectos en destino y origen: $5 \mathrm{ca}$ sos de estudio en países y economías en desarrollo. COFIDES, ESADE y ECONOMISTAS.

[8] NORBERG, J. (2017). Progreso. 10 razones para mirar el futuro con optimismo. Deusto e Instituto Juan de Mariana. 


\section{Información Comercial Española \\ Revista de Economía}

6 números anuales

Artículos originales sobre un amplio espectro de temas tratados desde

una óptica económica, con especial referencia a sus aspectos internacionales

Boletín Económico de Información Comercial Española

\section{2 números anuales}

Artículos y documentos sobre economía española, comunitaria e internacional, con especial énfasis en temas sectoriales y de comercio exterior
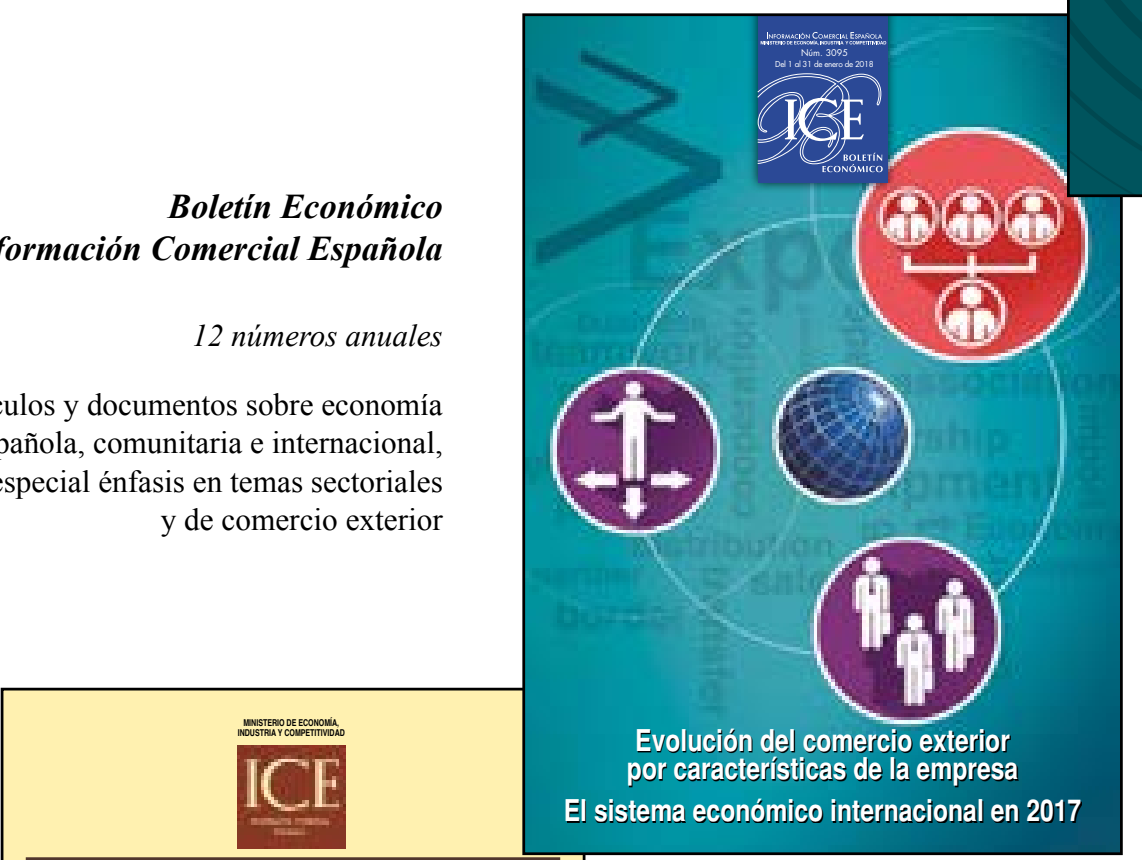

Efectos económicos

y empresariales de

los flujos migratorios
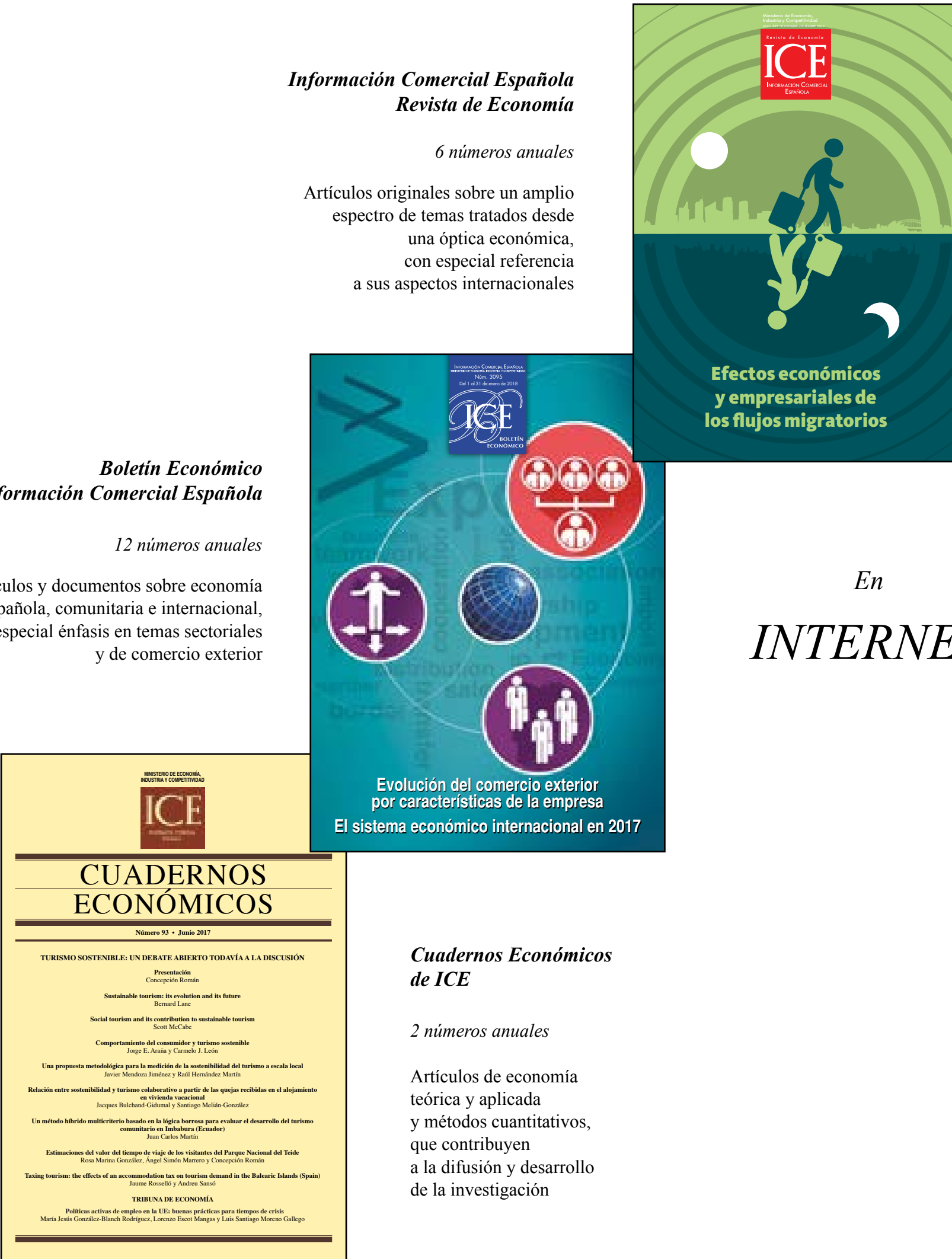

\section{Cuadernos Económicos de ICE}

2 números anuales

Artículos de economía teórica y aplicada y métodos cuantitativos, que contribuyen a la difusión y desarrollo de la investigación 